\title{
Diffusion- thermo and Thermo-diffusion Effects on Heat and Mass Transfer MHD Visco-elastic Flow Past a Plate
}

\author{
Rita Choudhury ${ }^{1}$ and Bibhash Deka ${ }^{2}$ \\ ${ }^{1,2}$ Department of Mathematics, Gauhati University, Guwahati-781014, Assam, India. \\ ${ }^{1}$ E-mail: rchoudhury66@yahoo.in \\ ${ }^{2}$ E-mail: bibhashdeka66@gmail.com
}

\begin{abstract}
The MHD free convective laminar flow of a visco-elastic fluid past over a vertical plate moving with uniform velocity in presence of Soret and Dufour effects has been analysed. The non-Newtonian fluid flow is characterized by second-grade model which follows from generalized Rivlin-Ericksen fluid. A uniform magnetic field is applied transversely to the direction of the main stream. Regular perturbation technique is used to solve the coupled non-linear differential equations. Approximate solutions for velocity, temperature, concentration and shearing stress at the plate have been derived. The rate of heat transfer and the rate of mass transfer in terms of Nusselt number and Sherwood number respectively are also obtained. Emphasis has been given on the effects of the visco-elasticity with the combination of other physical parameters involve in the solution. The results are illustrated graphically to observe the visco-elastic effects in possible cases. The applications of the present study have been applied in the fields of chemical engineering, hydrology and polymer technology.
\end{abstract}

2010 Mathematics Subject Classification : 76A05, 76A10

Keywords: MHD, Soret and Dufour effects, Heat and Mass Transfer, Radiation and Visco-elastic.

\section{INTRODUCTION}

The property of fluids which exhibits both viscous and elastic characteristics while undergoing deformation is known as viscoelasticity. For the last few decades, the study of MHD visco-elastic fluid flow over a vertical plate in presence of other fluid properties has attracted the researchers for its wide application in various fields like soil physics, engineering sciences, biological system, astrophysics, aeronautics etc. Also, the analysis of mass and heat transfer in flow problem is very important because of its vast applications in oil reservoir engineering and geothermal studies.

Soret and Dufour effects are exceptional physical phenomenon in the study of fluid mechanics. The heat transfer created by concentration gradient is known as diffusion thermo or Dufour effect. Again, the mass transfer caused by temperature gradient is termed as thermal-diffusion or Soret effect. Alam and Rahman investigated the Dufour and Soret effects on MHD free convective heat and mass transfer flow past a vertical flat plate embedded in porous medium [1]. Anghel et al. [2] studied the Dufour and Soret effects on free convective boundary layer over a vertical surface embedded in a porous medium. The Diffusion-thermo and thermaldiffusion effects in transient and steady natural convection from vertical surface has been studied by Dursunkaya and Worek [3]. Kafousias and Williams [4] have analysed the Thermal-diffusion diffusion- thermo effects on mixed free forced convective and mass transfer boundary layer flow with temperature dependent. The effect of chemical reaction and thermal stratification on MHD free convective heat and mass transfer over a vertical stretching surface embedded in a porous media considering Soret and Dufour number has been investigated by Mansour et al. [5]. Nazmul and Mahmud [6] have presented the Dufour and Soret effects on steady MHD free convection and mass transfer fluid flow through a porous medium in a rotating system. Influence of a magnetic field on heat and mass transfer by a natural convection from vertical surfaces in a porous media considering Soret and Dufour effects has been investigated by Postelincus [7]. Raptis and Kafousias [8] have presented the MHD free convection flow and mass transfer through porous medium bounded by an infinite vertical porous plate with constant heat flux. Sedeek [9] has studied the Thermal-diffusion and diffusion-thermo effects on mixed free-forced convective flow and mass transfer over accelerating surface with a heat source in the presence of suction and blowing in the case of variable viscosity. Soret effect on free convection and mass transfer flow in the stokes problem for an infinite vertical plate has been studied by Jha and Singh [10]. In the study of fluid flow problems, thermal radiation is also a very important physical phenomenon when the difference between the surface of a material and the surrounding temperature is large. Due to its practical applications, the thermal radiation problems has attracted the attention to several researchers for last few decades and is extensively studied to understand the same. Analytical study of magnetohydrodynamic radiation convection with surface temperature oscillation and secondary flow effects has been studied by Beg and Ghosh [11]. Ibrahim et al. [12] have presented the effects of the chemical reaction and 
radiation absorption on the unsteady MHD free convection flow past a semi-infinite vertical permeable moving plate with heat source and suction. Dufour and Soret effects of a transient free convection flow with radiative heat transfer past a flat plate moving through a binary mixture has been investigated by Oladapo [13]. Uwanta et al. [14] have analysed the MHD fluid flow over a vertical plate with Dufour and Soret effects.

The main objective of this paper is to analyse the effect of visco-elasticity on the MHD free convective incompressible flow of a second-grade fluid with heat and mass transfer in presence of Soret and Dufour effects. The constitutive equation for the second-order fluid is of the form,

$\sigma=-p I+\mu_{1} A_{1}+\mu_{2} A_{2}+\mu_{3} A_{1}^{2}$

where $\sigma$ is the stress tensor, $A_{n}(n=1,2)$ are the kinematic Rivlin-Ericksen tensors. $\mu_{i}(i=1,2,3)$ are the material coefficients describing the viscosity, elasticity and cross viscosity respectively. From thermodynamic considerations, it is noticed that the material coefficients $\mu_{1}$ and $\mu_{3}$ are positive and $\mu_{2}$ is negative [Coleman and Markovitz [15]]. The equation (1.1) was established by Coleman and Noll [16] from the simple fluids by assuming that stress is more sensitive to the recent deformation than to the deformation that occurred in the distant past. It is reported that solution of poly-isobutylene in cetane at $30^{\circ} \mathrm{C}$ simulate a second-grade fluid and the material constants for the solutions of various concentrations have been determined by Markovitz .

\section{MATHEMATICAL FORMULATION}

We consider unsteady MHD free convective incompressible laminar flow of a visco-elastic fluid past a moving vertical plate with Soret and Dufour effects in presence of heat and mass transfer. $X$-axis is taken along the length of the plate and $Y$-axis normal to it. Let the fluid is moving with velocity $U$ along $X$ direction. A magnetic field of uniform strength $B_{0}$ is applied transversely to the direction of the main flow. Assuming that the surface temperature is to oscillate with small amplitude about a non-uniform mean temperature.In this study, we consider that the magnetic Reynolds number is very very less than unity so that we can neglect the induced magnetic field in comparison with the applied magnetic field. Also, we assume that all the fluid properties except the density in the buoyancy force term are constant. Then, under the usual Boussines's approximation, the governing equations of mass, momentum, energy and concentration are as follows:

$\frac{\partial U}{\partial X}=0$

$\frac{\partial U}{\partial \bar{t}}=v_{1} \frac{\partial^{2} U}{\partial Y^{2}}+v_{2} \frac{\partial^{3} U}{\partial \bar{t} \partial Y^{2}}+g \beta\left(T-T_{\infty}\right)+g \beta^{*}\left(C-C_{\infty}\right)-\frac{\sigma B_{0}^{2}}{\rho} U$

$\frac{\partial T}{\partial \bar{t}}=\frac{k_{t}}{\rho c_{p}} \frac{\partial^{2} T}{\partial Y^{2}}+\frac{v_{1}}{c_{p}}\left(\frac{\partial U}{\partial Y}\right)^{2}+\frac{v_{2}}{c_{p}} \cdot \frac{\partial U}{\partial Y} \cdot \frac{\partial^{2} U}{\partial \bar{t} \partial Y}-\frac{1}{\rho c_{p}} \frac{\partial q_{r}}{\partial Y}+\frac{D_{m} k_{T}}{c_{s} c_{p}} \frac{\partial^{2} C}{\partial Y^{2}}$

$\frac{\partial C}{\partial \bar{t}}=D \frac{\partial^{2} C}{\partial Y^{2}}+\frac{D_{m} k_{T}}{T_{m}} \frac{\partial^{2} T}{\partial Y^{2}}$

The corresponding boundary conditions are:

$U=U_{w}, T=T_{w}+\varepsilon\left(T_{w}-T_{\infty}\right) e^{i \bar{\omega} \bar{t}}, C=C_{w}+\varepsilon\left(C_{w}-C_{\infty}\right) e^{i \bar{\omega} \bar{t}}$ at $Y=0$

$U \rightarrow 0, T \rightarrow 0, C \rightarrow 0$ in $Y \rightarrow \infty$

where $\bar{t}$ is the time, $v_{1}$ is the kinematic viscosity, $v_{2}$ is the visco-elasticity, $g$ is the acceleration due to gravity, $\beta$ is the coefficient of volumetric expansion for heat transfer, $\beta^{*}$ is the coefficient of volumetric expansion for mass transfer, $T$ is the fluid temperature, $T_{\infty}$ is the temperature of the fluid far away from the plate, $C$ is the species concentration, $C_{\infty}$ is the species concentration of the fluid far away from the plate, $\sigma$ is the electrical conductivity, $\rho$ is the fluid density, $k_{t}$ is the thermal conductivity, $c_{p}$ is the specific heat at constant pressure, $q_{r}$ is the radiative heat flux, $D_{m}$ is the coefficient of mass diffusivity, $c_{s}$ is the concentration susceptibility, $k_{T}$ is the thermal diffusion, $D$ is the molecular diffusivity, $T_{m}$ is the mean fluid temperature, $U_{w}$ is the velocity of the plate, $T_{w}$ is the temperature of the plate and $C_{w}$ is the concentration of the plate.

The thermal radiation flux gradient can be expressed as,

$-\frac{\partial q_{r}}{\partial Y}=4 a \sigma^{*}\left(T_{\infty}^{4}-T^{4}\right)$

where $a$ is the absorption coefficient and $\sigma^{*}$ is the Stefan - Boltzmann constant. Assuming that the differences in temperature within the flow are such that $T^{4}$ can be expressed as a linear combination of the temperature, we expand $T^{4}$ in Taylor series about $T_{\infty}$ and neglecting the higher- order terms we get,

$T^{4} \approx 4 T_{\infty}^{3} T-3 T_{\infty}^{4}$ 
We introduce the dimensionless quantities:

$u=\frac{U}{U_{w}}, y=\frac{Y U_{w}}{v_{1}}, t=\frac{\bar{t} U_{w}{ }^{2}}{v_{1}}, G r=\frac{g \beta v_{1}\left(T_{w}-T_{\infty}\right)}{U_{w}{ }^{3}}, M=\frac{\sigma B_{0}{ }^{2} v_{1}}{\rho U_{w}{ }^{2}}, D u=\frac{D_{m} k_{T}\left(C_{w}-C_{\infty}\right)}{c_{s} c_{p} v_{1}\left(T_{w}-T_{\infty}\right)}, K_{1}=\frac{16 a \sigma^{*} v_{1}{ }^{2} T_{\infty}^{3}}{k_{t} U_{w}{ }^{2}}, P r=$ $\frac{\rho v_{1} c_{p}}{k_{t}}, S c=\frac{v_{1}}{D}, S r=\frac{D_{m} k_{T}\left(T_{w}-T_{\infty}\right)}{T_{m} v_{1}\left(C_{w}-C_{\infty}\right)}, \theta=\frac{T-T_{\infty}}{T_{w}-T_{\infty}}, \phi=\frac{C-C_{\infty}}{C_{w}-C_{\infty}}, G c=\frac{g \beta^{*} v_{1}\left(C_{w}-C_{\infty}\right)}{U_{w}{ }^{3}}, E=\frac{U_{w}{ }^{2}}{\left(T_{w}-T_{\infty}\right)}, \omega=$

$\frac{\bar{\omega} v_{1}}{U_{w}^{2}}, \alpha_{1}=\frac{v_{2} U_{w}^{2}}{v_{1}^{2}}$

The equations (2.2) to (2.4) yield the following dimensionless equations with the use of the expressions (2.7) to (2.9):

$\frac{\partial u}{\partial t}=\frac{\partial^{2} u}{\partial y^{2}}+\alpha_{1} \frac{\partial^{3} u}{\partial t \partial y^{2}}+G r \theta+G c \phi-M u$

$\operatorname{Pr} \frac{\partial \theta}{\partial t}=\frac{\partial^{2} \theta}{\partial y^{2}}+\operatorname{PrE}\left\{\left(\frac{\partial u}{\partial y}\right)^{2}+\alpha_{1} \frac{\partial u}{\partial y} \cdot \frac{\partial^{2} u}{\partial t \partial y}\right\}-\operatorname{Pr} K_{1} \theta+\operatorname{Pr} D u \frac{\partial^{2} \phi}{\partial y^{2}}$

$S c \frac{\partial \phi}{\partial t}=\frac{\partial^{2} \phi}{\partial y^{2}}+\operatorname{SrSc} \frac{\partial^{2} \theta}{\partial y^{2}}$

The relevant boundary conditions are:

$u=1, \theta=1+\varepsilon e^{i \omega t}, \phi=1+\varepsilon e^{i \omega t}$ at $y=0$

$u \rightarrow 0, \theta \rightarrow 0, \phi \rightarrow 0$ in $y \rightarrow \infty$

where $y$ is the non-dimensional horizontal coordinate, $u$ is the dimensionless axial velocity, $t$ is the dimensionless time, $\theta$ is the dimensionless temperature, $\phi$ is the non-dimensional species concentration, $\operatorname{Pr}$ is the Prandtl number, $S c$ is the Schmidt number, $M$ is the Hartmann number, $G r$ is the thermal Grashof number, $G c$ is the solutal Grashof number, E is the Eckert number, $K_{1}$ is the thermal radiation parameter, $S r$ is the Soret number, $D u$ is the Dufour number, $\varepsilon$ is small reference parameter, $\omega$ is the frequency parameter and $\alpha_{1}$ is the visco-elastic parameter.

\section{METHOD of SOLUTION}

To solve the differential equations (2.10) to (2.12) subject to boundary conditions (2.13) and (2.14), we use perturbation technique and take the reference parameter $\varepsilon$ as perturbation parameter where $\varepsilon \ll 1$. Expanding $u, \theta$ and $\phi$ in powers of $\varepsilon$ and neglecting the higher order terms of $o\left(\varepsilon^{2}\right)$, we have

$u(y, t)=u_{0}(y)+\varepsilon u_{1}(y) e^{i \omega t}$
$\theta(y, t)=\theta_{0}(y)+\varepsilon \theta_{1}(y) e^{i \omega t}$
$\phi(y, t)=\phi_{0}(y)+\varepsilon \phi_{1}(y) e^{i \omega t}$

Substituting (3.1) to (3.3) in the equations (2.10) to (2.12) and equating the like powers of $\varepsilon$, we get the following equations:

Zeroth-order equations:

$u_{0}^{\prime \prime}-M u_{0}=-G r \theta_{0}-G c \phi_{0}$

$\theta_{0}^{\prime \prime}-\operatorname{Pr} K_{1} \theta_{0}=-\operatorname{Pr} E u_{0}^{\prime 2}-\operatorname{PrDu} \phi_{0}^{\prime \prime}$

$\phi_{0}^{\prime \prime}=-\operatorname{SrSc} \theta_{0}^{\prime \prime}$

First- order equations:

$\left(1+i \omega \alpha_{1}\right) u_{1}^{\prime \prime}-(M+i \omega) u_{1}=-G r \theta_{1}-G c \phi_{1}$

$\theta_{1}^{\prime \prime}+\operatorname{PrE} \alpha_{1} i \omega u_{1}^{\prime} u_{0}^{\prime}-\left(K_{1}+i \omega\right) \operatorname{Pr} \theta_{1}=-2 \operatorname{PrE} u_{0}^{\prime} u_{1}^{\prime}-\operatorname{PrDu} \phi_{1}^{\prime \prime}$

$\phi_{1}^{\prime \prime}-i \omega S c \phi_{1}=-\operatorname{Sr} S c \theta_{1}^{\prime \prime}$

The corresponding boundary conditions are :

$u_{0}=1, u_{1}=0 ; \theta_{0}=1=\theta_{1} ; \phi_{0}=1=\phi_{1}$ at $y=0$

$u_{0} \rightarrow 0, u_{1} \rightarrow 0 ; \theta_{0} \rightarrow 0, \theta_{1} \rightarrow 0 ; \phi_{0} \rightarrow 0, \phi_{1} \rightarrow 0$ in $y \rightarrow \infty$

The equations (3.4) to (3.9) are still coupled for the variables $u_{0}, \theta_{0}, \phi_{0}, u_{1}, \theta_{1}$ and $\phi_{1}$. To solve these differential equations with the boundary conditions (3.10) and (3.13), we take the Eckert number $E$ as perturbation parameter since Eckert number is very small for incompressible fluids. Expanding $u_{0}, \theta_{0}, \phi_{0}, u_{1}, \theta_{1}$ and $\phi_{1}$ in powers of the Eckert number $E$ and neglecting the higher order terms of $o\left(E^{2}\right)$, we have

$u_{0}(y)=u_{00}(y)+E u_{01}(y)$

$\theta_{0}(y)=\theta_{00}(y)+E \theta_{01}(y)$

$\phi_{0}(y)=\phi_{00}(y)+E \phi_{01}(y)$

$u_{1}(y)=u_{10}(y)+E u_{11}(y)$ 
$\theta_{1}(y)=\theta_{10}(y)+E \theta_{11}(y)$

$\phi_{1}(y)=\phi_{10}(y)+E \phi_{11}(y)$

Substituting (3.12) to (3.17) in the equations (3.4) to (3.9) and equating the like powers of $E$, we get the following equations:

Zeroth-order equations:

$$
\begin{aligned}
& u_{00}^{\prime \prime}-M u_{00}=-\operatorname{Gr} \theta_{00}-G c \phi_{00} \\
& \theta_{00}^{\prime \prime}-\operatorname{Pr} K_{1} \theta_{00}=-\operatorname{PrDu} \phi_{00}^{\prime \prime} \\
& \phi_{00}^{\prime \prime}=-\operatorname{Sr} S c \theta_{00}^{\prime \prime} \\
& \left(1+i \omega \alpha_{1}\right) u_{10}^{\prime \prime}-(M+i \omega) u_{10}=-\operatorname{Gr} \theta_{10}-G c \phi_{10} \\
& \theta_{10}^{\prime \prime}-\left(K_{1}+i \omega\right) \operatorname{Pr} \theta_{10}=-\operatorname{PrD} u \phi_{10}^{\prime \prime} \\
& \phi_{10}^{\prime \prime}-i \omega S c \phi_{10}=-\operatorname{SrSc} \theta_{10}^{\prime \prime}
\end{aligned}
$$

First- order equations:

$u_{01}^{\prime \prime}-M u_{01}=-G r \theta_{01}-G c \phi_{01}$

$\theta_{01}^{\prime \prime}-\operatorname{Pr} K_{1} \theta_{01}=-\operatorname{Pr} \cdot u_{00}^{\prime 2}-\operatorname{PrDu} \phi_{01}^{\prime \prime}$

$\phi_{01}^{\prime \prime}=-\operatorname{SrSc} \theta_{01}^{\prime \prime}$

$\left(1+i \omega \alpha_{1}\right) u_{11}^{\prime \prime}-(M+i \omega) u_{11}=-G r \theta_{11}-G c \phi_{11}$

$\theta_{11}^{\prime \prime}+\operatorname{Pr}_{1} i \omega u_{10}^{\prime} u_{00}^{\prime}-\left(K_{1}+i \omega\right) \operatorname{Pr} \theta_{11}=-2 \operatorname{Pr} u_{00}^{\prime} u_{10}^{\prime}-\operatorname{PrD} u \phi_{11}^{\prime \prime}$

$\phi_{11}^{\prime \prime}-i \omega S c \phi_{11}=-\operatorname{SrSc} \theta_{11}^{\prime \prime}$

with boundary conditions,

$u_{00}=1, u_{01}=0, u_{10}=0=u_{11} ; \theta_{00}=1, \theta_{01}=0, \theta_{10}=1, \theta_{11}=0 ; \phi_{00}=1, \phi_{01}=0, \phi_{10}=1, \phi_{11}=$ 0 at $y=0$

$u_{00} \rightarrow 0, u_{01} \rightarrow 0, u_{10} \rightarrow 0, u_{11} \rightarrow 0 ; \theta_{00} \rightarrow 0, \theta_{01} \rightarrow 0, \theta_{10} \rightarrow 0, \theta_{11} \rightarrow 0 ; \phi_{00} \rightarrow 0, \phi_{01} \rightarrow 0, \phi_{10} \rightarrow 0, \phi_{11} \rightarrow$ 0 in $y \rightarrow \infty$

Solving the equations (3.18) to (3.29) subject to the boundary conditions (3.30) and (3.31), we get

$\phi_{00}=-S r . S c \cdot e^{-\sqrt{A_{1}} y}+1+S r . S c$

$\theta_{00}=e^{-\sqrt{A_{1}} y}$

$u_{00}=A_{2} e^{-\sqrt{M} y}-A_{3} e^{-\sqrt{A_{1}} y}+\frac{G c(1+S r . S c)}{M}$

$\phi_{01}=S r . S c \cdot \frac{\operatorname{Pr} .\left(A_{4}-A_{5}-A_{6}\right)}{1-\text { Pr.Du.Sr.Sc }} e^{-\sqrt{A_{1}} y}-S r . S c \cdot \frac{P r}{1-\text { Pr.Du.Sr.Sc }}\left\{A_{4} e^{-\left(\sqrt{M}+\sqrt{A_{1}}\right) y}-A_{5} e^{-2 \sqrt{A_{1}} y}-A_{6} e^{-2 \sqrt{M} y}\right\}$

$\theta_{01}=\frac{P r}{1-P r . D u . S r . S c}\left(A_{6}+A_{5}-A_{4}\right) e^{-\sqrt{A_{1}} y}+\frac{P r}{1-\text { Pr.Du.Sr.SC }}\left\{A_{4} e^{-\left(\sqrt{M}+\sqrt{A_{1}}\right) y}-A_{5} e^{-2 \sqrt{A_{1}} y}-A_{6} e^{-2 \sqrt{M} y}\right\}$

$u_{01}=\left[-A_{7}+A_{8}\left\{\frac{A_{4}}{\left(\sqrt{M}+\sqrt{A_{1}}\right)^{2}-M}-\frac{A_{5}}{4 A_{1}-M}-\frac{A_{6}}{3 M}\right\}+A_{9}-A\left\{\frac{A_{4}}{\left(\sqrt{M}+\sqrt{A_{1}}\right)^{2}-M}-\frac{A_{5}}{4 A_{1}-M}-\frac{A_{6}}{3 M}\right\}\right] e^{-\sqrt{M} y}-$

$A_{8}\left\{\frac{A_{4}}{\left(\sqrt{M}+\sqrt{A_{1}}\right)^{2}-M} e^{-\left(\sqrt{M}+\sqrt{A_{1}}\right) y}-\frac{A_{5}}{4 A_{1}-M} e^{-2 \sqrt{A_{1}} y}-\frac{A_{6}}{3 M} e^{-2 \sqrt{M} y}\right\}-A_{9} e^{-\sqrt{A_{1}} y}+$

$A_{10}\left\{\frac{A_{4}}{\left(\sqrt{M}+\sqrt{A_{1}}\right)^{2}-M} e^{-\left(\sqrt{M}+\sqrt{A_{1}}\right) y}-\frac{A_{5}}{4 A_{1}-M} e^{-2 \sqrt{A_{1}} y}-\frac{A_{6}}{3 M} e^{-2 \sqrt{M} y}\right\}$

$\phi_{10}=e^{-\sqrt{\frac{b_{1}+\sqrt{b_{1}^{2}-4 b_{2}}}{2} y}}$

$\theta_{10}=\left(1+A_{11}\right) e^{-\sqrt{\left(K_{1}+i \omega\right) P r}} y-A_{11} e^{-\sqrt{\frac{b_{1}+\sqrt{b_{1}^{2}-4 b_{2}}}{2}} y}$

$u_{10}=A_{14} e^{-\sqrt{\frac{M+i \omega}{1+i \omega \alpha_{1}}} y}-\frac{G r A_{11}}{1+i \omega \alpha_{1}}\left\{A_{12} e^{-\sqrt{\left(K_{1}+i \omega\right) P r} y}-A_{13} e^{-\sqrt{\frac{b_{1}+\sqrt{b_{1}^{2}-4 b_{2}}}{2} y}}\right\}-\frac{G c A_{13}}{1+i \omega \alpha_{1}} e^{-\sqrt{\frac{b_{1}+\sqrt{b_{1}^{2}-4 b_{2}}}{2} y}}$

$\phi_{11}=A_{35} e^{-\sqrt{\frac{b_{1}+\sqrt{b_{1}^{2}-4 b_{2}}}{2} y}}+A_{29} e^{-A_{23} y}-A_{30} e^{-A_{24} y}+A_{31} e^{-A_{25} y}-A_{32} e^{-A_{26} y}+A_{33} e^{-A_{27} y}+A_{34} e^{-A_{28} y}$ 


$$
\begin{aligned}
& \theta_{11}=\frac{1}{S r S c}\left\{A_{43}-A_{36} e^{-\sqrt{\frac{b_{1}+\sqrt{b_{1}^{2}-4 b_{2}}}{2} y}}-A_{37} e^{-A_{23} y}+A_{38} e^{-A_{24} y}-A_{39} e^{-A_{25} y}+A_{40} e^{-A_{26} y}-A_{41} e^{-A_{27} y}-\right. \\
& \left.A_{42} e^{-A_{28} y}\right\} \\
& u_{11}=A_{52} e^{-\sqrt{\frac{M+i \omega}{1+i \omega \alpha_{1}}} y}-\frac{1}{1+i \omega \alpha_{1}}\left\{A_{44} e^{-\sqrt{\frac{b_{1}+\sqrt{b_{1}^{2}-4 b_{2}}}{2}} y}+A_{45} e^{-A_{23} y}+A_{46} e^{-A_{24} y}+A_{47} e^{-A_{25} y}+A_{48} e^{-A_{26} y}+\right. \\
& \left.A_{49} e^{-A_{27} y}+A_{50} e^{-A_{28} y}-A_{51}\right\}
\end{aligned}
$$

The constants are not presented here due to sake of brevity. Now substituting the expressions of $u_{00}, u_{01}, u_{10}$, $u_{11}, \theta_{00}, \theta_{01}, \theta_{10}, \theta_{11}, \phi_{00}, \phi_{01}, \phi_{10}$ and $\phi_{11}$ in (3.1) to (3.3), the velocity, temperature and concentration of the problem are obtained.

\section{RESULTS and DISCUSSION}

Some significant flow characteristics of the problem viz. wall shear stress, local heat flux and mass flux are discussed below:

The non-dimensional shearing stress $\tau$ at the plate $y=0$ is given by

$\left.\tau=\frac{\partial u}{\partial y}+\alpha_{1} \frac{\partial^{2} u}{\partial t \partial y}\right]_{y=0}=A_{3} \sqrt{A_{1}}-A_{2} \sqrt{M}+E\left(A_{53}-A_{7} \sqrt{A_{1}}-A_{8} A_{54}+A_{9} \sqrt{A_{1}}+A_{10} A_{54}\right)+\varepsilon e^{i \omega t}(1+$ $\left.\alpha_{1} i \omega\right)\left(A_{55}+E A_{56}\right)$

The non-dimensional heat flux at the plate $y=0$ in terms of Nusselt number $N u$ is given by

$\left.N u=\frac{\partial \theta}{\partial y}\right]_{y=0}=-\sqrt{A_{1}}+E A_{57}+\varepsilon\left\{-A_{11} \sqrt{\left(K_{1}+i \omega\right) P r}+A_{11} \sqrt{\frac{b_{1}+\sqrt{b_{1}^{2}-4 b_{2}}}{2}}+E A_{58}\right\} e^{i \omega t}$

The non-dimensional mass flux at the plate $y=0$ in terms of Sherwood number $S h$ is given by

$\left.S h=\frac{\partial \phi}{\partial y}\right]_{y=0}=S r . S c \sqrt{A_{1}}+E A_{59}+\varepsilon\left\{-\sqrt{\frac{b_{1}+\sqrt{b_{1}^{2}-4 b_{2}}}{2}}+E A_{60}\right\} e^{i \omega t}$

To get the physical impact of the problem, the numerical computations have been executed for velocity, temperature and concentration fields. During the computations, we use different values of the visco-elastic parameter $\alpha_{1}$, magnetic parameter $\mathrm{M}$, Soret number $\mathrm{Sr}$, Dufour number $\mathrm{Du}$, thermal radiation parameter $K_{1}$, thermal Grashof number $G r$, solutal Grashof number $G c$ with fixed values of $\operatorname{Pr}=0.5, \mathrm{E}=.01, S c=0.9$, $\omega t=\frac{\pi}{2}, \omega=1$ and $\varepsilon=0.01$. Throughout the computation, only the real part of the problem has been considered. The purpose of this study is to bring out the effects of visco-elastic parameter $\alpha_{1}$ on the governing flow. The corresponding results for Newtonian fluid can be deduced from the above results by setting $\alpha_{1}=0$.

Figures 1 to 7 represent the first-order fluid velocity $u_{1}$ against $y$ for different values of other flow parameters. The graphs display that the fluid velocity enhances significantly near the plate but gradually lessens for both Newtonian and visco-elastic (non-Newtonian) fluids. Also, the first order fluid velocity accelerates with the growth of the absolute values of the visco-elastic parameter $\alpha_{1}\left(\alpha_{1}=0,-0.2,-0.4\right)$ in comparison with Newtonian fluid flow phenomenon. The rising values of magnetic parameter $M$ decelerate the fluid velocity in both system of fluids (Figs: 1and 2). The magnetic parameter represents the importance of magnetic field on the fluid as it flows vertically upward past a plate. The presence of transverse magnetic field yields Lorentz force which is a retarding force acts on the velocity profile. Therefore as magnetic parameter increases, so does the retarding force and this account for the decrease in fluid velocity. The rising values of the Soret number $\mathrm{Sr}$ (Figs: 1 and 3), Dufour number $D u$ (Figs: 1 and 4) and the thermal radiation parameter $K_{1}$ (Figs: 1 and 5) depict a decelerating trend of first- order fluid velocity but an opposite behaviour is experienced in fluid velocity during the growth of thermal Grashof number Gr (Figs: 1 and 6) and solutal Grashof number Gc (Figs: 1 and 7). The growth of radiation parameter implies the release of heat energy from the fluid flow region and so the motion of the fluid decelerates as the momentum boundary layer thickness becomes thinner. Again, with the enhancement 
of thermal and solutal Grashof numbers lead to the increase in thermal and mass buoyancy force in the boundary layer, as a result the velocity increases for both types of fluids.

The variation of shearing stress $\tau$ against the magnetic parameter $M$, Soret number $S r$, Dufour number $D u$, thermal Grashof number $G r$ and the solutal Grashof number $G c$ are displayed by the figures 8 to 12 respectively with fixed values of other physical parameters as mentioned. It is observed that the shearing stress enhances with the growth of absolute value of visco-elastic parameter $\alpha_{1}$ against $M, S r, D u$ and $G r$ but opposite trend is noticed against $G c$. Again, the growth of $S r, G r$ and $G c$ increase the viscous drag formed by Newtonian and visco-elastic fluids at the plate but an opposite result is noticed during the growing behaviour of $M$ in both the fluid flow systems. Also the shearing stress neither increases nor decreases with the enhancement of Dufour number $D u$ for both type of fluids.

The graphical representation of the first-order temperature $\theta_{1}$ and concentration $\phi_{1}$ are given in figures 13 and 14 respectively. From the figures it has been observed that the temperature and the concentration fields are not significantly affected by the visco-elastic parameter $\alpha_{1}$. The same statement is true for Nusselt number Nu and Sherwood number Sh.

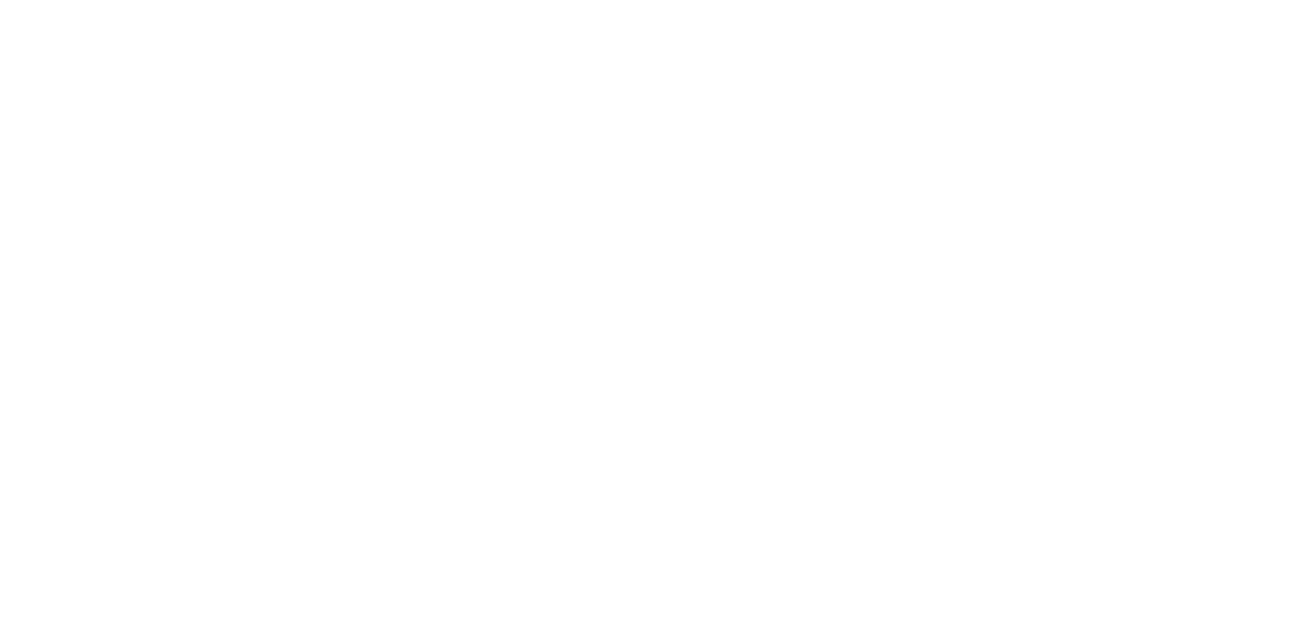

Fig 1: Variation of $u_{1}$ against $y$ for $M=1.5, S r=0.6, D u=0.8, K_{1}=0.6, G r=0.7$ and $G c=0.8$.

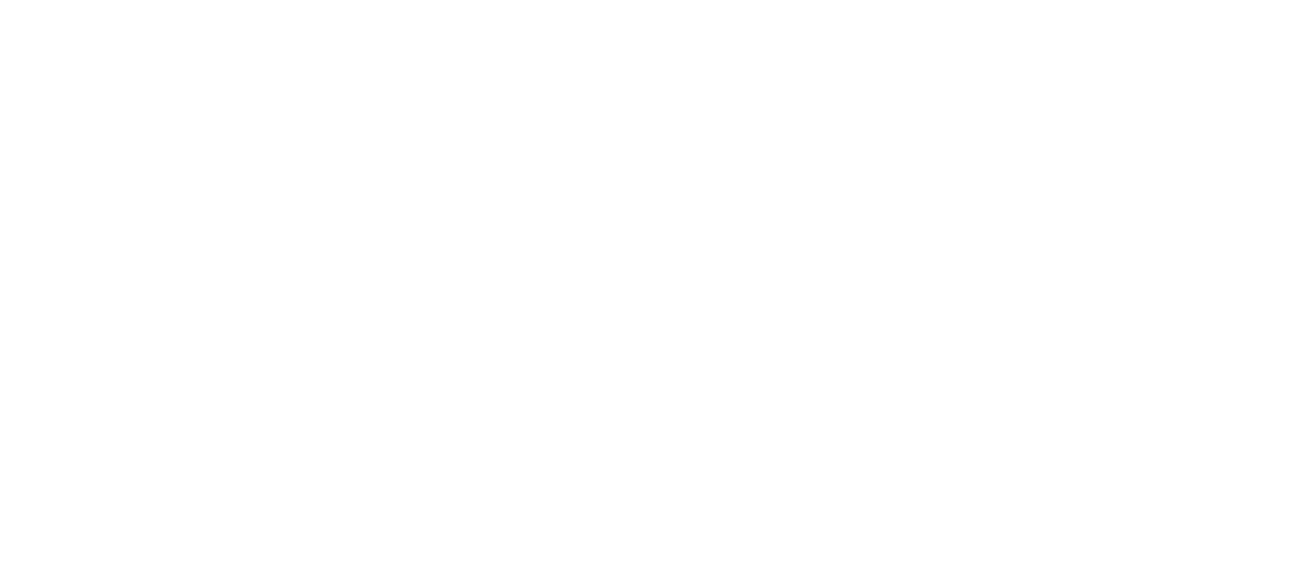

Fig 2: Variation of $u_{1}$ against $y$ for $M=2, S r=0.6, D u=0.8, K_{1}=0.6, G r=0.7$ and $G c=0.8$. 


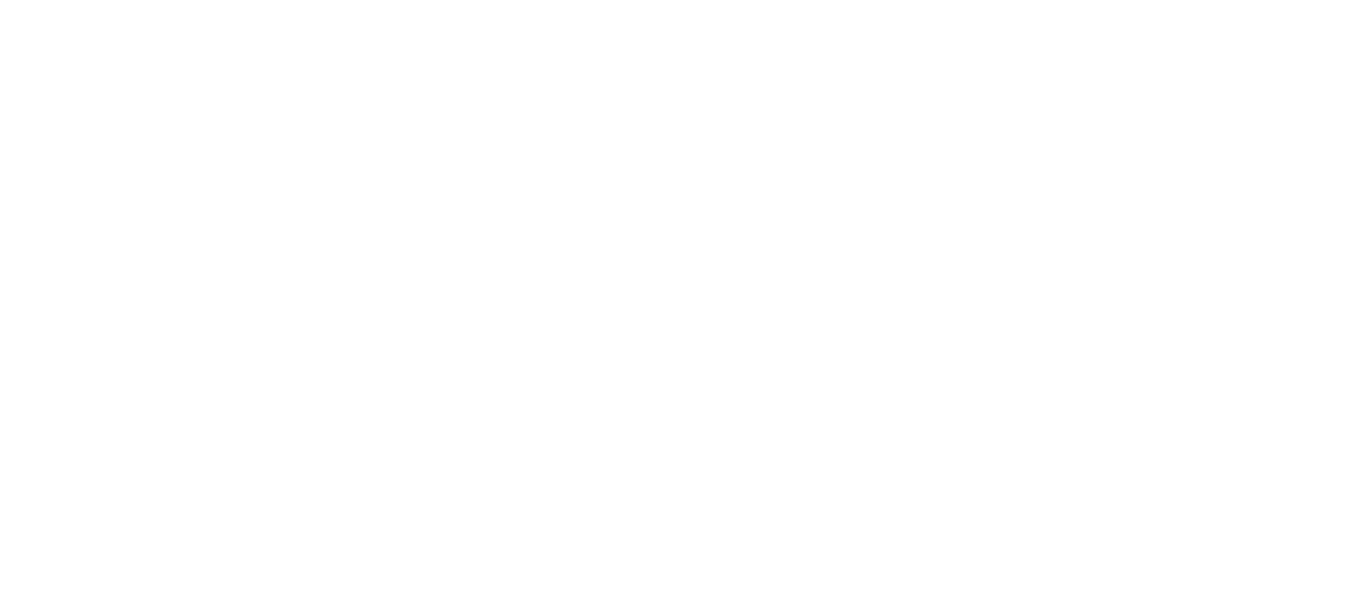

Fig 3: Variation of $u_{1}$ against $y$ for $M=1.5, S r=1.1, D u=0.8, K_{1}=0.6, G r=0.7$ and $G c=0.8$.

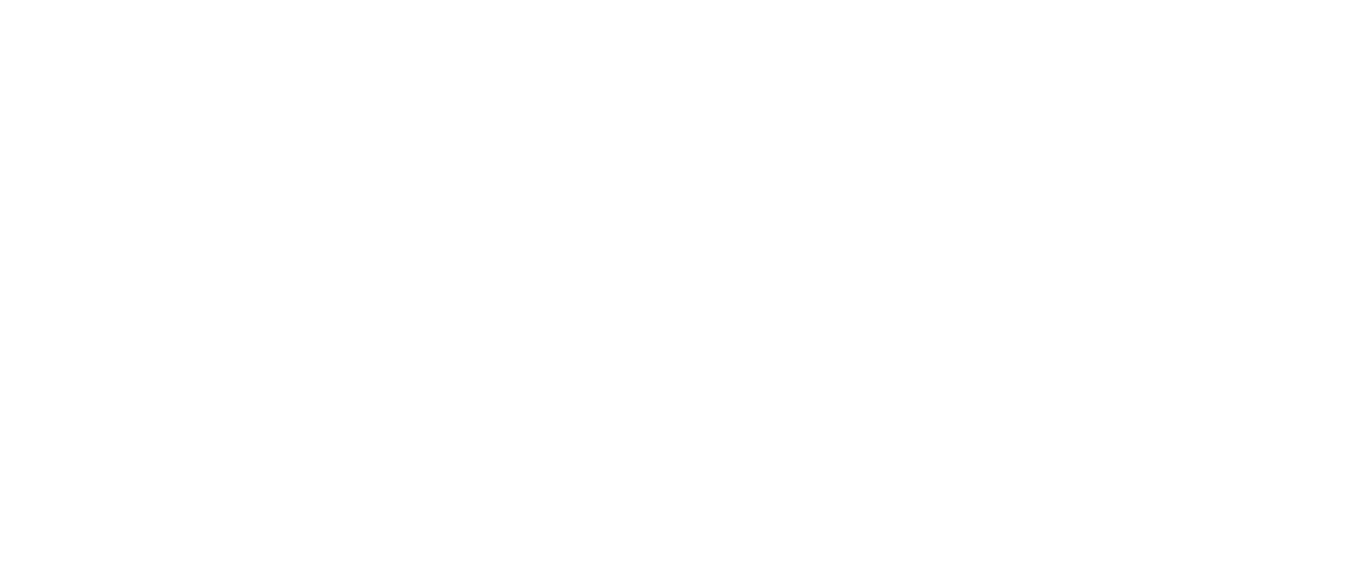

Fig 4: Variation of $u_{1}$ against $y$ for $M=1.5, S r=0.6, D u=1.3, K_{1}=0.6, G r=0.7$ and $G c=0.8$.

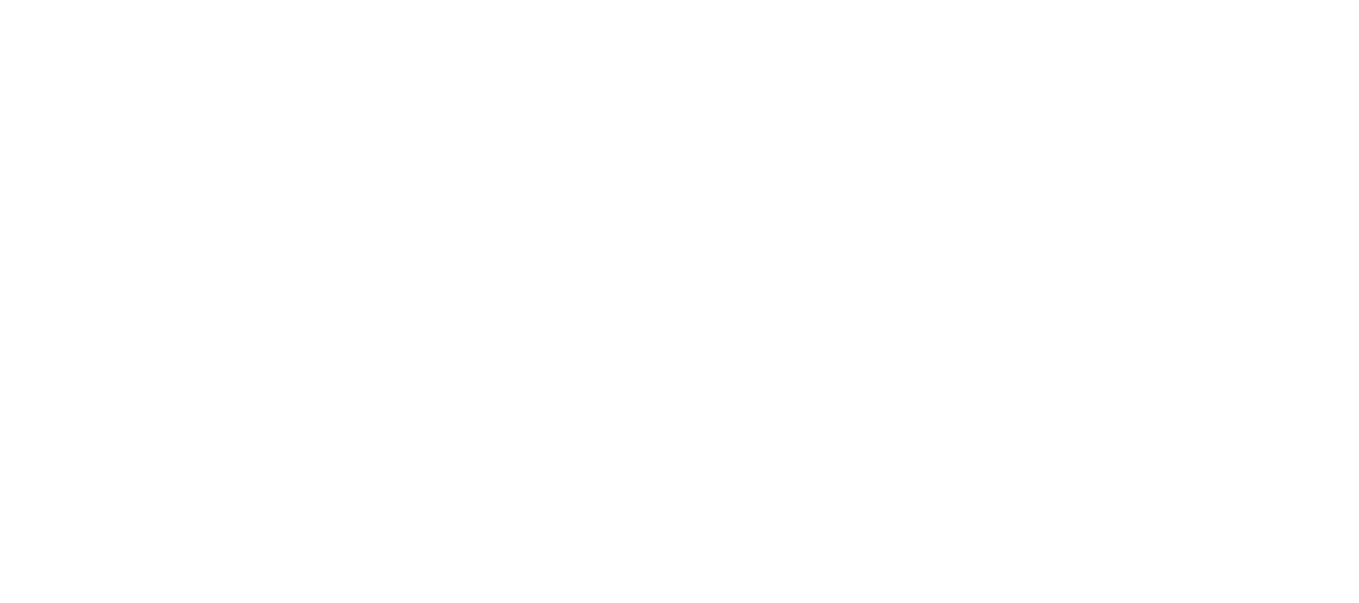

Fig 5: Variation of $u_{1}$ against $y$ for $M=1.5, S r=0.6, D u=0.8, K_{1}=1.1, G r=0.7$ and $G c=0.8$. 


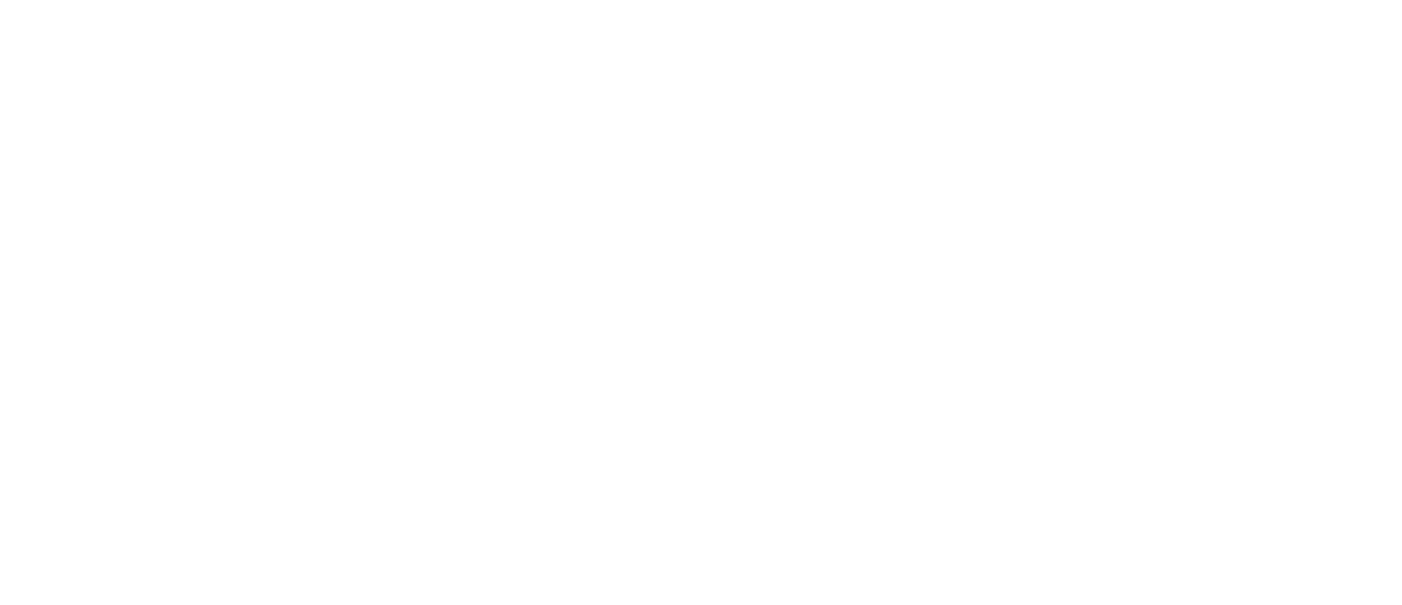

Fig 6: Variation of $u_{1}$ against $y$ for $M=1.5, S r=0.6, D u=0.8, K_{1}=0.6, G r=1.2$ and $G c=0.8$.

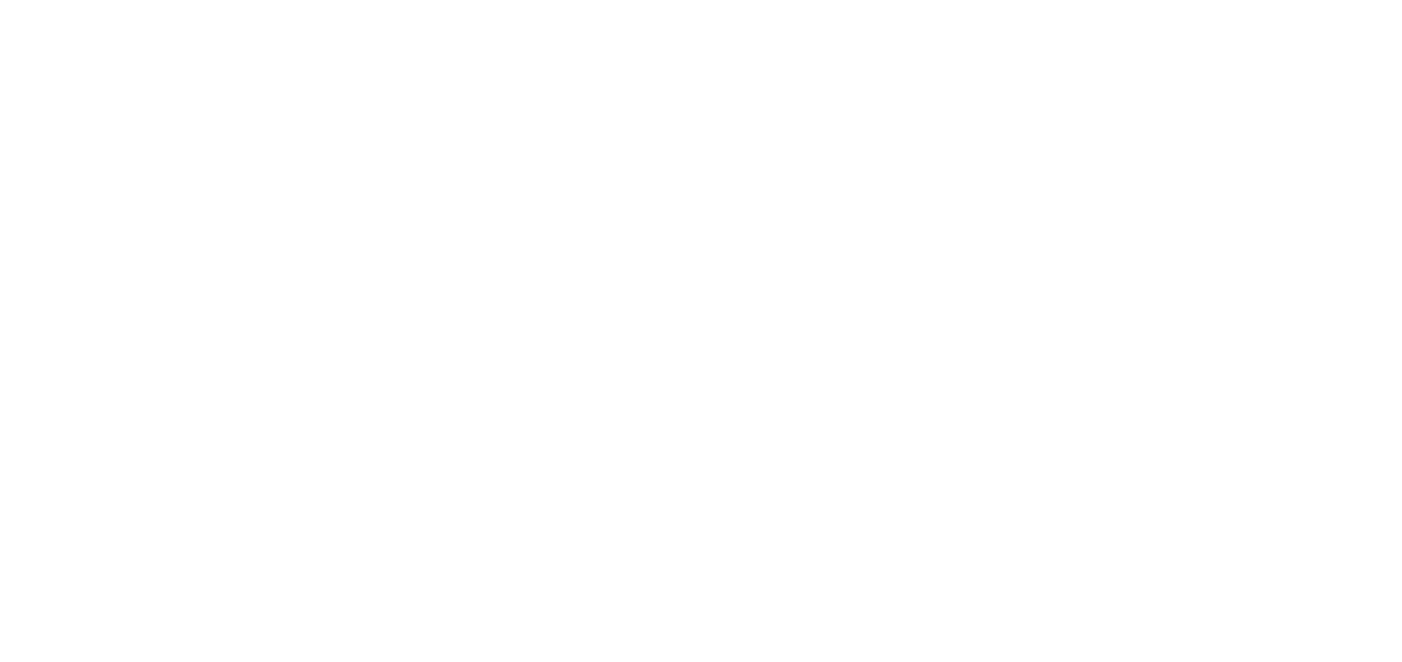

Fig 7: Variation of $u_{1}$ against $y$ for $M=1.5, S r=0.6, D u=0.8, K_{1}=0.6, G r=0.7$ and $G c=1.3$.

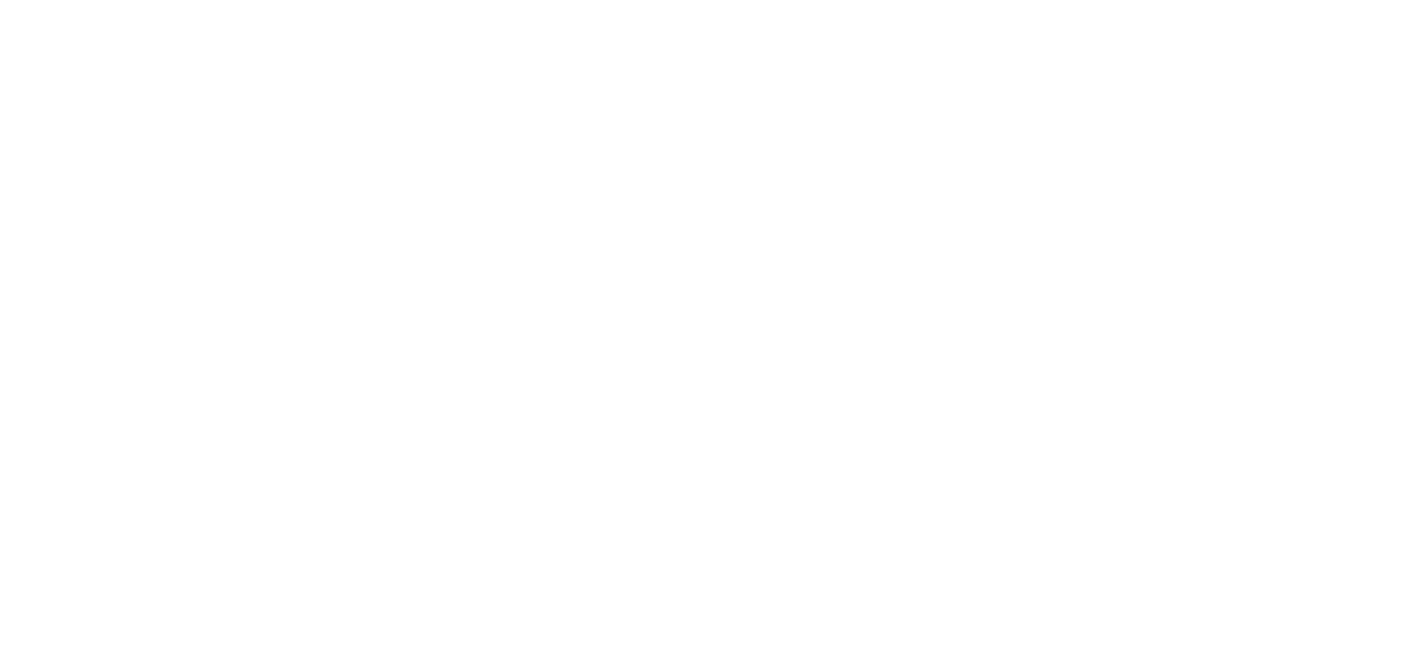

Fig 8: Variation of $\tau$ against $M$ for $S r=0.6, D u=0.8, K_{1}=0.6, G r=0.7$ and $G c=1.3$. 


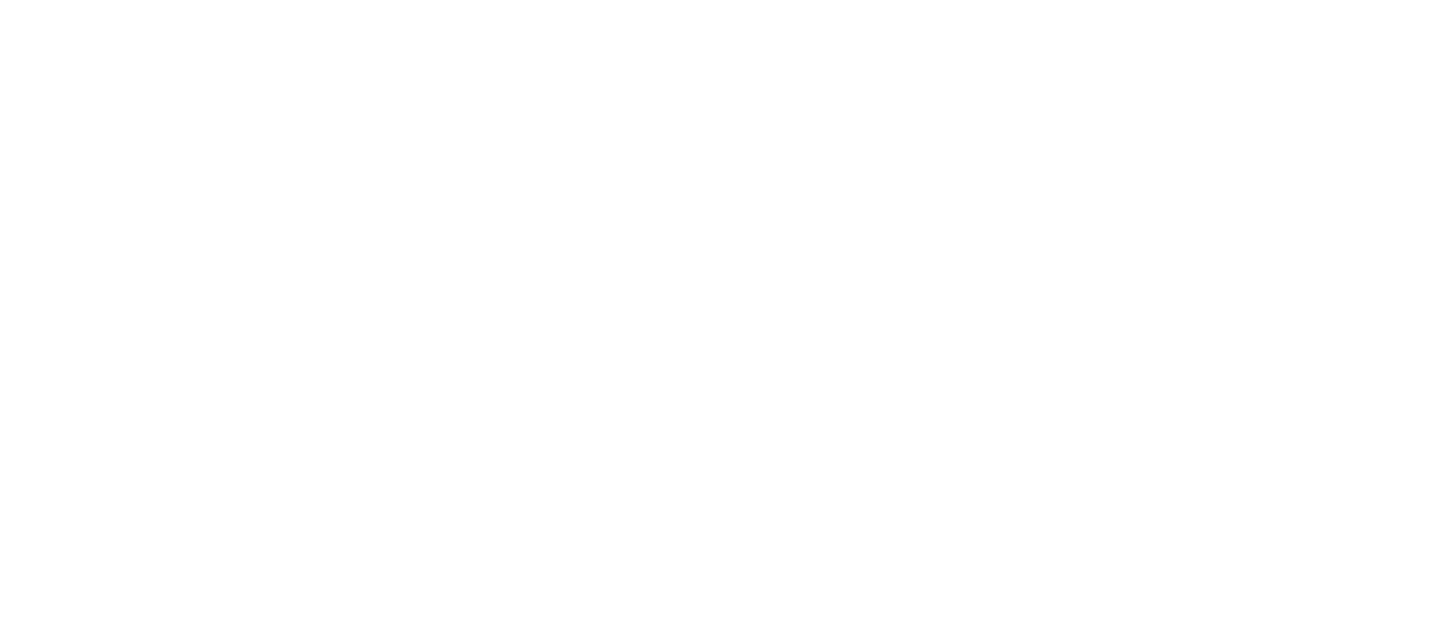

Fig 9: Variation of $\tau$ against $S r$ for $M=1.5, D u=0.8, K_{1}=0.6, G r=0.7$ and $G c=1.3$.

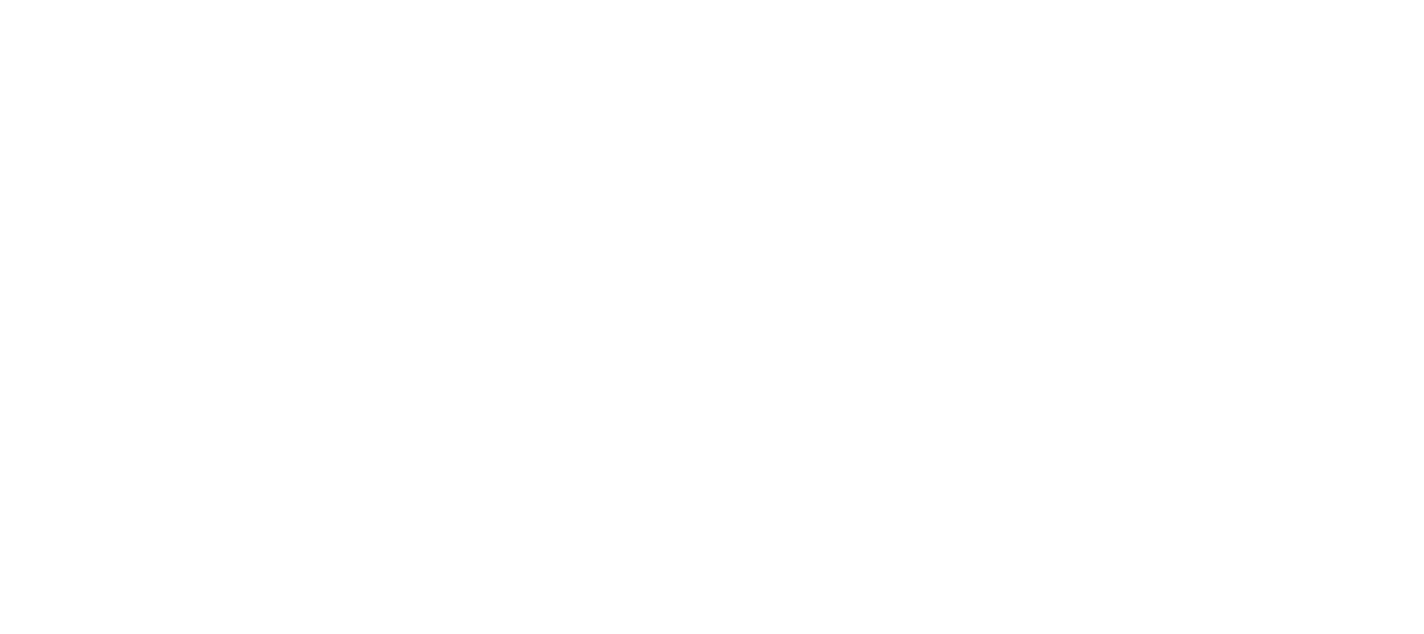

Fig 10: Variation of $\tau$ against $D u$ for $M=1.5, S r=0.6, K_{1}=0.6, G r=0.7$ and $G c=1.3$

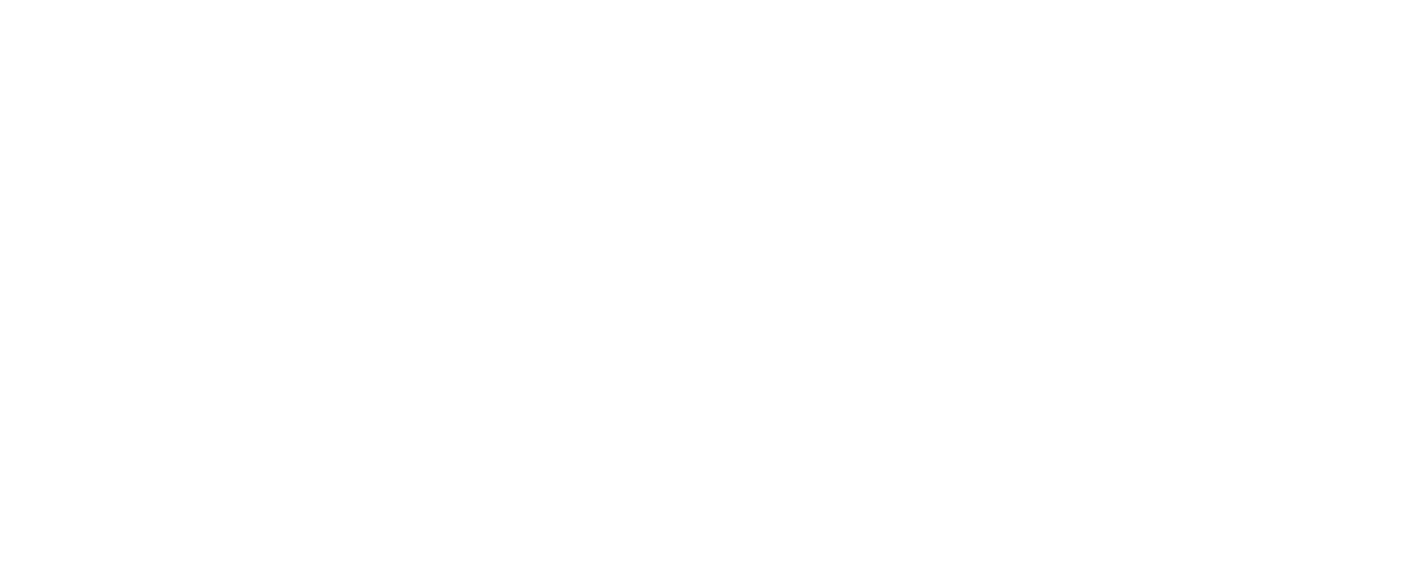

Fig 11: Variation of $\tau$ against $G r$ for $M=1.5, S r=0.6, K_{1}=0.6, D u=0.8$ and $G c=1.3$. 


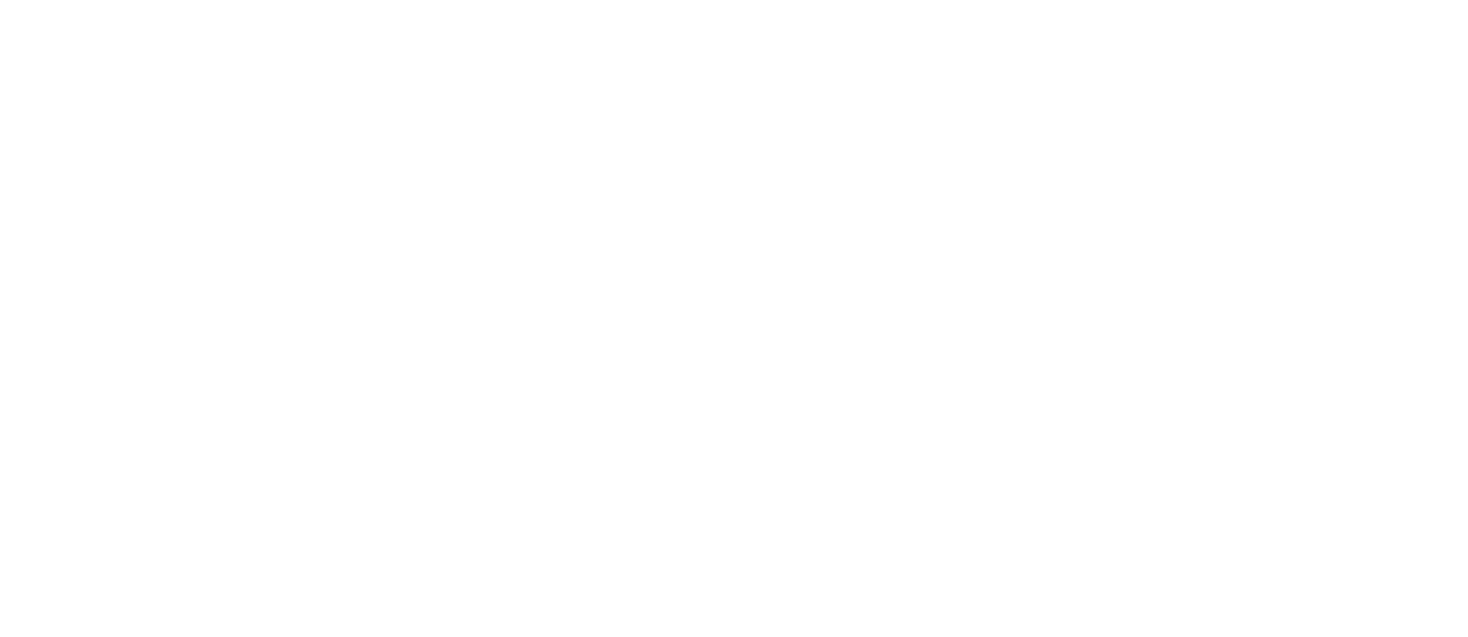

Fig 12: Variation of $\tau$ against $G c$ for $M=1.5, S r=0.6, K_{1}=0.6, G r=0.7$ and $D u=0.8$.

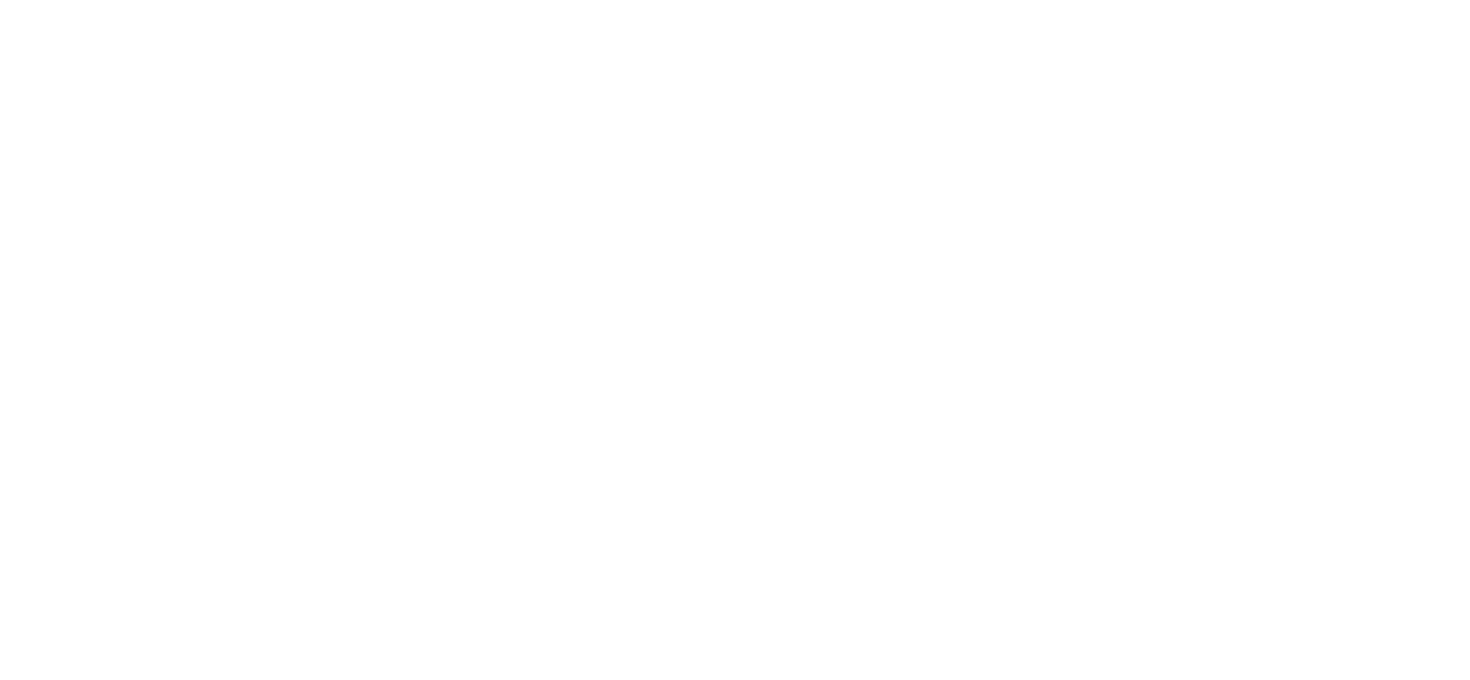

Fig 13: Variation of $\theta_{1}$ against $y$ for $M=1.5, S r=0.6, D u=0.8, K_{1}=0.6, G r=0.7$ and $G c=0.8$.

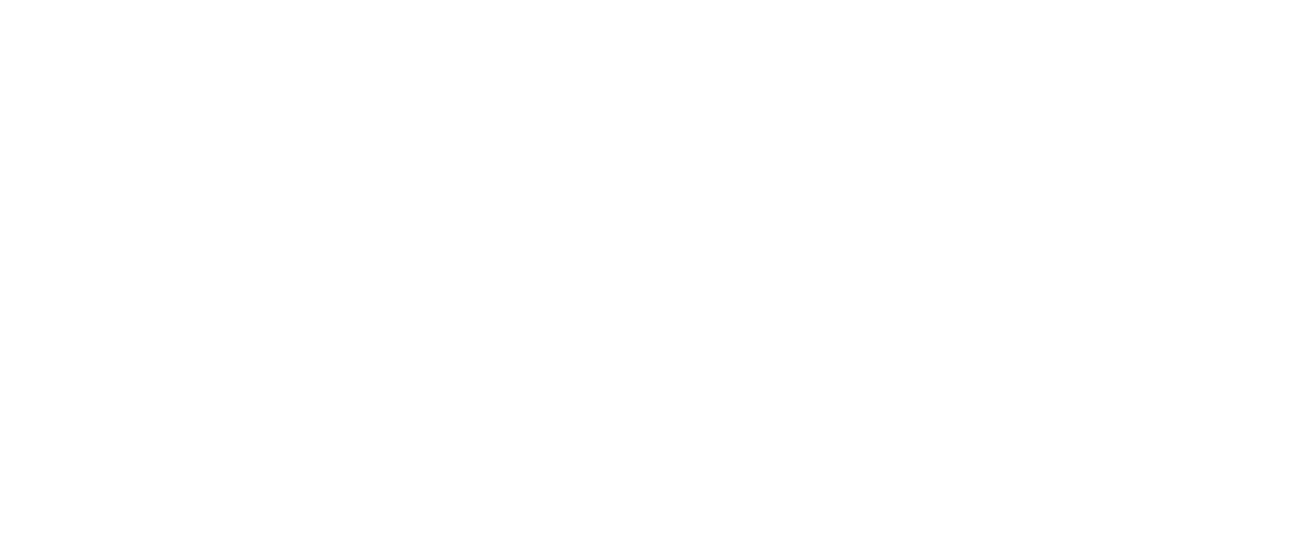

Fig 14: Variation of $\phi_{1}$ against $y$ for $M=1.5, S r=0.6, D u=0.8, K_{1}=0.6, G r=0.7$ and $G c=0.8$. 


\section{CONCLUSIONS}

The study leads to the following conclusions:

- The first-order velocity field is significantly affected at each point of the flow region by the visco-elastic parameter $\alpha_{1}$.

- The enhancement of absolute value of visco-elasticity depicts the accelerating trend of first-order fluid velocity in comparison with Newtonian fluid flow phenomenon.

- The enhancement of magnetic parameter decelerates the fluid velocity which shows conformity with physical situation.

- The growth of Soret number diminishes the fluid velocity and this trend is noticed in case of Dufour number and radiation parameter.

- With the growth of different important flow parameters, the shearing stress affects significantly by both Newtonian and non-Newtonian cases.

- The first-order temperature and concentration profiles are not considerably affected by the visco-elastic parameter. This is because of restraining effect played by the elasticity of the fluid.

- There is no significant influence of visco-elastic parameter on Nusselt number and Sherwood number.

\section{ACKNOWLEDGEMENT}

The financial support by UGC is gratefully acknowledged by the authors.

\section{REFERENCES}

[1] Alam, M.S., and Rahman, M.M., 2005, "Dufour and Soret effects on MHD free convective heat and nass transfer flow past a vertical flat plate embedded in porous medium”, J. NavalArch. and Marine Engg., vol.2, pp.55-65.

[2] Anghel, M., Takhar, H.S., and Pop, I., 2000, "Dufour and Soret effects on free convective boundary layer over a vertical surface embedded in a porous medium”, J. of Heat and Mass Trans., vol. 43, pp.1265-1274.

[3] Dursunkaya, Z., and Worek, W.M., 1992, "Diffusion thermo and thermal-diffusion effects in transient and steady natural convection from vertical surface”, Int. J. Heat and Mass Trans, vol. 35, pp.2060-2065.

[4] Kafousias, N.G., and Williams, E.M., 1995, “Thermal-diffusion diffusion- thermo effects on mixed free forced convective and mass transfer boundary layer flow with temperature dependent”, Int. J. of Engg.Sci., vol.33, pp.1369-1384.

[5] Mansour, M.A., El-Anssary, N.F., and Aly, A.M., 2008 "Effect of chemical reaction and thermal stratification on MHD free convective heat and mass transfer over a vertical stretching surface embedded in a porous media considering Soret and Dufour number”, Chem. Engg.J., vol. 145, pp.340-345.

[6] Nazmul, I., and Mahmud, A., 2007, "Dufour and Soret effects on steady MHD free convection and mass transfer fluid flow through a porous medium in a rotating system”, J.ofNaval Arch. and Marine Engg., vol. 4, pp.43-55.

[7] Postelincus, A., 2004, "Influence of a magnetic field on heat and mass transfer by a natural convection from vertical surfaces in a porous media considering Soret and Dufour effects”, Int. J. of Heat and Mass Trans., vol.47, pp.1467-1472.

[8] Raptis, A., and Kafousias, N.G., 1982, "MHD free convection flow and mass transfer through porous medium bounded by an infinite vertical porous plate with constant heat flux, Cambridge J. Phys., vol. 60, pp.1725-1729.

[9] Sedeek, M.A., 2004, "Thermal-diffusion and diffusion-thermo effects on mixed free-forced convective flow and mass transfer over accelerating surface with a heat source in the presence of suction and blowing in the case of variable viscosity”, Acta. Mechanica, vol. 172, pp.83-94.

[10] Jha, B.K., and Singh, A.K., 1990, "Soret effect on free convection and mass transfer flow in the stokes problem for an infinite vertical plate”, Astrophys and Space Sci., vol.173, pp.251-255.

[11] Beg, O.A., and Ghosh, S.K., 2010, “Analytical study ofmagnetohydrodynamic radiation convection with surface temperature oscillation and secondary flow effects”, Int. J. of Appl. Math. and Mech., vol. 6, pp.1-22.

[12] Ibrahim, F.S., Elaiw, A.M., and Bakr, A.A., 2008, "Effects of the chemical reaction and radiation absorption on the unsteady MHD free convection flow past a semi-infinite vertical permeable moving plate with heat source and suction”, Cambridge J. Phys., vol. 78, pp.255-270.

[13] Oladapo, P.O., 2010, "Dufour and Soret effects of a transient free convection flow withradiative heat transfer past a flat plate moving through a binary mixture”, Pacific J. of Sci. and Tech., vol. 11, pp.163-172.

[14] Uwanta, I.J., Asogwa, K.K., and Ali, U.A., 2012, "MHD fluid flow over a vertical plate with Dufour and Soret effects", Int. J. of Computer Applications, vol. 45, pp.8-16.

[15] Coleman, B.D., and Markovitz, H., 1964, “Incompressible second-order fluids”, Adv. Appl. Mech, vol. 8, pp.69-101.

[16] Coleman, B.D., and Noll,W., 1960, “An applications theorem for functional with applications in continuum mechanics”, Archs Ration Mech. Analysis, vol.6,pp.350-360. 